AN. MED. INTERNA (Madrid) Vol. 18, N. ${ }^{\circ} 8$, pp. 411-414, 2001

\title{
Cetoacidosis diabética en un departamento de Urgencias
}

\author{
A. SEGADO SORIANO, M J. GRANDA MARTÍN, C. LÓPEZ GONZÁLEZ-COBOS, \\ M. V. VILLALBA GARCÍA, M. GÓMEZ ANTÚNEZ, J. GIL GÓMEZ, A. MUIÑO \\ MÍGUEZ \\ Departamento de Urgencias. Hospital General Universitario Gregorio Marañón. Madrid
}

\author{
KETOACIDOSIS DIABETIC IN AN EMERGENCY DEPARTMENT
}

\section{RESUMEN}

Objetivos: Estudiamos las características clínicas y las diferencias existentes en los pacientes con Cetoacidosis Diabética (CAD) atendidos en un Departamento de Urgencias.

Métodos: Describimos las características de 15 pacientes con criterios de CAD durante un periodo de 12 meses. Evaluamos prospectivamente: características clínicas, factores precipitantes, enfermedades concomitantes, conocimiento previo o no de la diabetes, complicaciones, mortalidad y necesidad de ingreso en la Unidad de Cuidados Intensivos (UCI). Comparamos a los mismos según dos características: grupos de edad y diagnóstico previo o no de diabetes mellitus.

Resultados: La CAD es más frecuente en mujeres jóvenes. La mortalidad de pacientes con CAD está directamente relacionada con la edad y con procesos concomitantes, siendo más elevada en ancianos.El factor precipitante más frecuente en pacientes con diagnóstico nuevo de diabetes eran los procesos infecciosos y entre los diabéticos conocidos era el abandono del tratamiento.

Conclusiones: La mortalidad de los pacientes con CAD se relaciona con la edad y procesos concomitantes. Hay diferencias claras en cuanto a factores precipitantes de la CAD según sean diabéticos conocidos o no.

PALABRAS CLAVE: Cetoacidosis diabética. Grupos de edad. Mortalidad.

\begin{abstract}
Objective: We study clinical data and differences in the patients with Diabetic Ketoacidosis (CAD) atended in an Emergency Department (ED).

Methods: We described 15 patients with criteria for diagnosis of $C A D$ during a 12-month period. We value prospectively clinical data precipitating factors, diagnosis associated, previous diagnosed diabetes, complications, mortality and use of and intensive care unit (UCI). We comparing groups according two characteristics: age group and prior diagnosis of diabetes mellitus.
\end{abstract}

KEY WORDS: Ketoacidosis diabetic. Age group. Mortality.

Segado Soriano A, Granda Martín MJ, López González-Cobos C, Villalba García MV, Gómez Antúnez M, Gil Gómez J, Muiño Míguez A. Cetoacidosis diabética en un departamento de Urgencias. An Med Interna (Madrid) 2001; 18: 411-414.

\section{INTRODUCCIÓN}

La diabetes hoy día continúa siendo una enfermedad con una obvia morbilidad y mortalidad asociada, los diabéticos realizan más consultas médicas ambulatorias, requieren más ingresos hospitalarios, con estancias más prolongadas, que los no diabéticos. La cetoacidosis diabética (CAD) es la complicación aguda típica de la diabetes tipo I ,que en ocasiones también aparece en diabéticos tipo II. Fue descrita por Ara- teus de Capadocia en el 200 a.d. C. Roger Unger la define como un desorden bihormonal caracterizado por niveles bajos de insulina y altos de glucagón. Este disbalance pone en marcha una serie de mecanismos que implican a las células hepáticas y grasas desencadenando los efectos clínicos de la CAD. La mortalidad oscila entre 5-10\% según las series. En personas de edades superiores a 60 años la mortalidad puede alcanzar el $20 \%$. Describimos las características clínicas y analíticas de 15 pacientes con criterios de CAD (Fig. 1), que

Trabajo aceptado: 12 de Marzo de 2001

Correspondencia: A. Segado Soriano. Pasaje José Sánchez Pescador 4, 5-A. 28007 Madrid 


\begin{tabular}{l|l} 
Mayores & Menores \\
\hline $\mathrm{pH}<7,30$ & Glucemia $>200 \mathrm{mg} / \mathrm{dl}$ \\
Bicabornato $<15 \mathrm{meq} / \mathrm{l}$ & Anión GAP elevado \\
Cetoácidos plasma $>3 \mathrm{mg} / \mathrm{dl}$ &
\end{tabular}

Fig. 1. Criterios de cetoacidosis diabética (CAD). Se considera CAD si cumple 3 criterios mayores o 2 mayores y 1 menor.

ingresaron de forma consecutiva en nuestro Departamento de Urgencias durante el periodo comprendido entre el 1 junio 1998 y el 31 mayo 1999. Describimos las diferencias existentes según grupos de edad. Además valoramos las diferencias existentes entre diabéticos conocidos y no conocidos (CAD como primera manifestación de la misma).

\section{MÉTODOS}

El Hospital General Universitario Gregorio Marañón es un centro hospitalario de tercer nivel, que abarca un área poblacional aproximada de 630.000 habitantes. Valoramos de forma prospectiva todos los pacientes que acudieron de forma consecutiva a nuestro Departamento de Urgencias (Sección de Medicina) durante el periodo comprendido entre el 1 de junio de 1998 y el 31 mayo de 1999 seleccionando aquellos que cumplían criterios de CAD (15 pacientes). Excluimos los atendidos por Cirugía, Traumatología y Ginecología, así como los menores de 16 años. Analizamos en los 15 pacientes características demográficas, antecedentes personales, factor desencadenante, conocimiento previo de su diabetes, diagnósticos concomitantes, síntomas y signos al ingreso, pruebas complementarias seriadas (al ingreso, 2, 6, 12, 18 y 24 horas), evolución y necesidad de ingreso en la Unidad de Cuidados Intensivos (UCI). Nuestros objetivos fueron: describir características generales de la serie, estudiar las diferencias existentes según grupo de edad (diferenciando 3 grupos: < 30 años, 30-65 años y >65 años), y por otro lado analizar las dife- rencias entre los diabéticos conocidos y no conocidos (debut con CAD). El estudio estadístico se realizó utilizando la prueba del chi cuadrado, la " $t$ " de Student y el análisis de la varianza.

\section{RESULTADOS}

Las características generales de nuestra serie quedan reflejadas en la tabla I. La edad media era de $69 \pm 5$ años con predominio de mujeres $(11 / 15,73 \%)$. No encontramos diferencias estacionales. Los factores desencadenantes por orden de frecuencia fueron: patología infecciosa $(5 / 15,33 \%)$, incumplimiento del tratamiento $(4 / 15,26 \%)$, de origen desconocido en 3 pacientes $(20 \%)$. Sólo en el $10 \%$ hubo dos factores precipitantes simultáneos. El inicio fue gradual en el $60 \%$ de los pacientes. La triada clásica (sed, poliuria y polidipsia) se presentó en el $100 \%$ de los pacientes, seguido en orden de frecuencia por síntomas gastrointestinales (náuseas, vómitos, dolor abdominal) en el $60 \%$, disminución del nivel de conciencia y fiebre en el $33 \%$ respectivamente. Entre los signos clínicos destacaba el característico aliento cetósico $(73 \%)$, seguido de la deshidratación (66\%) . En UCI ingresaron 5/15 (33\%) falleciendo dos pacientes de nuestra serie (13\%), ambos en las primeras 18 horas tras el ingreso en urgencias.

Se distinguían tres grupos de edad, con evidentes diferencias entre ellos en cuanto a factor desencadenante y evolución (Tabla II): un primer grupo, inferior proporcionalmente al de otras series $(6 / 15,40 \%)$ de pacientes menores de 30 años, un segundo grupo de edad adulta (31-65 años) que proporcionalmente es superior a otras series $(6 / 15,40 \%)$, y un tercer grupo muy escaso en otras series pero no despreciable en la nuestra, de pacientes mayores de 65 años $(3 / 15,20 \%)$. En el grupo de menores de 30 años la CAD fue la primera manifestación conocida de su diabetes en el $50 \%$, la presentación fue gradual $(50 \%)$ y brusca $(50 \%)$, sólo 2 pacientes $(33 \%)$ precisaron ingreso en UCI. El principal factor desencadenante fue la aparición de un proceso infeccioso, destacando la influencia de la toxicomanía de base como desencadenante asociado a incumplimiento o a proceso infeccioso. No había predominio de

\section{TABLA I}

\section{CARACTERÍSTICAS GENERALES}

\begin{tabular}{|c|c|c|c|c|c|c|c|c|c|}
\hline Pacientes & Edad & Sexo & D.C. & F.D. & Inicio & $\mathrm{UCl}$ & Fiebre & Cetosis & Exitus \\
\hline $\mathrm{N} . \underline{0} 1$ & 82 & $M$ & No & I. mesentérica & Gradual & No & No & Sí & Sí \\
\hline $\mathrm{N}, 02$ & 60 & M & No & Desconocido & Gradual & No & No & Sí & No \\
\hline $\mathrm{N} .03$ & 18 & M & Sí & Inclumpimiento+Drogas & Brusco & Sí & No & Sí & No \\
\hline $\mathrm{N} . \underline{0} 4$ & 83 & M & Sí & I. arterial & Brusco & Sí & No & No & No \\
\hline N. 05 & 20 & M & No & Neumonía & Gradual & Sí & Sí & Sí & No \\
\hline N. 06 & 38 & $M$ & No & Desconocido & Brusco & Sí & Sí & Sí & No \\
\hline $\mathrm{N} . \underline{0} 7$ & 18 & $M$ & No & SDE febril+Drogas & Gradual & No & Sí & Sí & No \\
\hline N. 08 & 28 & $M$ & Sí & GEA & Brusco & No & No & Sí & No \\
\hline$N .09$ & 29 & $M$ & No & $\mathrm{GEA}$ & Gradual & No & Sí & Sí & No \\
\hline N. 010 & 62 & $M$ & Sí & Inclumpimiento & Brusco & No & No & No & No \\
\hline N. 011 & 45 & $M$ & Sí & Inclumpimiento & Gradual & No & No & No & No \\
\hline N. 012 & 45 & $M$ & Sí & Inclumpimiento & Gradual & No & No & Sí & No \\
\hline N. .13 & 57 & M & No & Neumonía & Gradual & Sí & Sí & Sí & No \\
\hline N.․ 14 & 24 & M & Sí & Desconocido & Brusco & No & No & Sí & No \\
\hline$N .015$ & 85 & $M$ & Sí & Pancreatitis & Gradual & No & No & Sí & Sí \\
\hline
\end{tabular}

D.C. = Diabetes conocida; F.D. = Factor desencadenante 


\section{TABLA II}

\section{DIFERENCIAS EN CAD SEGÚN GRUPOS EDAD}

\begin{tabular}{lccc}
\hline Grupos de edad & $<30$ años & $30-65$ años & $>65$ años \\
\hline N.. pacientes & $6 / 15(40 \%)$ & $6 / 15(40 \%)$ & $3 / 15(20 \%)$ \\
Sexo & 50\%, M 50\% & $\vee 17 \%, M$ M $83 \%$ & $M 100 \%$ \\
Inicio gradual & $50 \%$ & $66 \%$ & $66 \%$ \\
Inicio brusco & $50 \%$ & $34 \%$ & $34 \%$ \\
Debut diabético & $50 \%$ & $33 \%$ & $33 \%$ \\
Infeccioso* & $66 \%$ & $16 \%$ & $0 \%$ \\
Incumplimiento* & $66 \%$ & $50 \%$ & $0 \%$ \\
Desconocido* & $16 \%$ & $33 \%$ & $0 \%$ \\
Otros* & $0 \%$ & $0 \%$ & $100 \%$ \\
UCl & $33 \%$ & $16 \%$ & $33 \%$ \\
Exitus & $0 \%$ & $0 \%$ & $66 \%$ \\
\hline
\end{tabular}

Varón $=$ Varón; $M=$ M ujer; * = Factor desencadenante

ningún sexo (50\% de varones y 50\% de mujeres) y la mortalidad fue nula. En el grupo de edad intermedia la diabetes era conocida en el $66 \%$,el inicio fue gradual de forma predominante $(66 \%)$, con presencia mayoritaria de mujeres $(5 / 6$, $83 \%$ ), sólo 1 paciente de este grupo de edad ingresó en UCI. En este grupo el principal factor desencadenante fue el incumplimiento del tratamiento (50\%), seguido de causas desconocidas $(33 \%)$, siendo la mortalidad nula. En el grupo de edad superior a 65 años el inicio fue gradual en $2 / 3$ pacientes, y es en este grupo donde se centra la mortalidad de nuestras serie $(2 / 3,66 \%)$, con presencia en los tres pacientes de procesos concomitantes graves: en los fallecidos: pancreatitis con criterios de gravedad e isquemia mesentérica respectivamente, en el superviviente la presencia de isquemia arterial de extremidades inferiores. Dos pacientes ingresaron en UCI. La mortalidad se produjo en las primeras 18 horas, y se relaciona de forma significativa con la presencia de glucemias $>700 \mathrm{mg} / \mathrm{dl}$, de $\mathrm{pH}<7$ y cuerpos cetónicos en sangre $>3 \mu \mathrm{g} / \mathrm{dl}$.

Por otro lado estudiamos las diferencias clínicas y analíticas entre dos grupos de nuestra serie: grupo A o de diabéticos conocidos (8/15) y grupo B (7/15) de diabéticos no conocidos (CAD como primera manifestación de diabetes) (Tabla III).

En el grupo A el tiempo desde su diagnóstico era muy

\section{TABLA III}

\section{DIFERENCIAS EN CAD SEGÚN DIABETES PREVIA}

\begin{tabular}{lcc}
\hline Grupos & Grupo A & Grupo B \\
\hline N.o pacientes & 8 & 7 \\
Varones & $1 / 8(12,5 \%)$ & $3 / 7(42,8 \%)$ \\
M ujeres & $7 / 8(87,5 \%)$ & $4 / 7(57,2 \%)$ \\
Inicio gradul & $3 / 8(37,5 \%)$ & $6 / 7(85 \%)$ \\
Inicio brusco & $5 / 8(62,5 \%)$ & $1 / 7(15 \%)$ \\
HTA* & $6 / 8(75 \%)$ & $1 / 7(15 \%)$ \\
C. isquémica* & $1 / 8(12,5 \%)$ & $0 / 7(0 \%)$ \\
ACVA* & $2 / 8(25 \%)$ & $0 / 7(0 \%)$ \\
Infeccioso+ & $1 / 8(12,5 \%)$ & $4 / 7(57,2 \%)$ \\
Desconocido+ & $1 / 8(12,5 \%)$ & $2 / 7(27,8 \%)$ \\
Otros+ & $2 / 8(25 \%)$ & $1 / 7(15 \%)$ \\
\hline
\end{tabular}

Grupo A = D.M . previa; Grupo B = D.M . no conocida; * = Antecedentes; $+=$ Desencadenante variable, entre 3-20 años, con claro predominio de mujeres $(7 / 8,87 \%)$, destacando la elevada proporción en ellos de otros factores de riesgo cardiovascular sobre todo HTA $(6 / 8,75 \%)$. En este grupo el factor desencadenante más frecuente era el incumplimiento del tratamiento $(50 \%)$, mientras que sólo en uno de ellos la CAD se desencadenó por causa infecciosa. El inicio fue brusco (síntomas de aparición en menos de 48 horas) en el $62 \%$ con disminución del nivel de conciencia y cifras de bicarbonato $<7,20$ en dichos casos. En el grupo B el factor desencadenante más frecuente fue un proceso infeccioso $(57 \%)$, principalmente neumonías, siendo el inicio en la mayoría de los casos gradual (síntomas de duración superior a 48 horas) $(6 / 7,85 \%)$

La principal complicación fue la hipoglucemia secundaria al tratamiento con bomba de insulina, y en los dos pacientes fallecidos fueron la trombosis mesentérica y la pancreatitis necrotizante. Ningún paciente presentó otras complicaciones clásicas descritas en la literatura como edema agudo de pulmón, edema cerebral...

Con respecto a la correlación $\mathrm{pH}$, bicarbonato, cuerpos cetónicos desde que se inicia el tratamiento, sólo en dos casos se observó una normalización del $\mathrm{pH}$ antes que los otros dos parámetros. La determinación de cuerpos cetónicos en orina no aportó información valida en el seguimiento terapéutico, pues como ya es conocido debido al método empleado del nitroprusiato, estos cuerpos cetónicos persisten positivos bastantes horas después de la normalización de los restantes parámetros. Tampoco observamos que la determinación de cuerpos cetónicos en plasma aportara mayor información en el seguimiento terapéutico en urgencias que el $\mathrm{pH}$, bicarbonato y glucemia en sangre, salvo su utilidad como criterio mayor de CAD.

Se obtuvieron hemocultivos en $7 / 15$ pacientes, siendo en todos ellos negativos, y, urocultivo en $6 / 15$ pacientes, siendo positivo para $E$. coli en 2 de ellos.

\section{DISCUSIÓN}

En nuestra serie hay varios hechos a destacar en comparación con otras de la literatura

La edad media descrita por otros autores es sensiblemente inferior: 33 años en la serie de May y cols. (1), 35 años en la de Snorgaard y cols. (2), mientras que en la nuestra es de 69 años, además en otras series hay un predominio de mujeres en todos los grupos de edad, mientras que en nuestra no encontramos esta diferencia en el grupo de edad < 30 años (1-6).

Con respecto a los factores precipitantes nuestra serie coincide con otras en cuanto a que los dos principales son las infecciones y el abandono del tratamiento, sin embargo en la nuestra hubo un menor porcentaje de dos factores o más desencadenantes simultáneos, con cifras del $10 \%$ mientras que en la literatura son del 20-30\% (1-4).Tampoco el alcohol aparecía como factor precipitante en ningún caso, a diferencia de lo descrito por May y cols. (1), donde se acerca al 25\%, sin embargo si observamos una importante relación de la aparición de CAD con la presencia de toxicomanía previa unida a otros factores desencadenantes en jóvenes.

Las diferencias clínicas entre aquellos con diabetes conocida y entre los que la CAD supuso la primera manifestación de dicha enfermedad fueron claras: en cuanto a factores desencadenantes, con predominio del incumplimiento entre 
los diabéticos conocidos y de las causas infecciosas entre los no conocidos, en cuanto a la forma de inicio, siendo predominantemente brusca entre los primeros y gradual entre los segundos. En el grupo de diabéticos conocido predominan las mujeres, lo cual relacionan algunos autores (6-11) con una mayor susceptibilidad en dicho sexo a la CAD.

Otra aportación no descrita en la literatura son las diferencias encontradas según el grupo de edad: el grupo de jóvenes es comparable a otras series en cuanto a factores desencadenantes, necesidad de ingreso en UCI en menos de la mitad, diferenciándose de otras series en la igualdad entre varones y mujeres, ya que en este grupo de edad son estas las mayoritarias en la mayoría de las series. En el grupo de edad media destacamos el predominio de mujeres, el incumplimiento del tratamiento como factor desencadenante predominante, así como la escasa presencia de procesos infecciosos. En los ancianos a diferencia de otras series destaca una elevadísima mortalidad, la relación de la misma con procesos concomitantes graves y cifras extremas de glucemia y $\mathrm{pH}$. Esto no lo describen otros autores, que relacionan la mortalidad con cifras de bicarbonato inferiores a $15 \mathrm{mg} / \mathrm{dl}$ e hipotensión al ingreso (1). En nuestra serie no se dieron complicaciones clásicas de la CAD como edema agudo de pulmón, edema cerebral, hipopotasemia, hiperamilasemia... lo cual podría explicarse por dos razones: inicio gradual en la mayoría, y, por un correcto seguimiento y tratamiento en el Departamento de Urgencias y en la UCI (11-14).

Las infecciones se asocian con mayor frecuencia a los pacientes con diabetes mellitus que acuden al Departamento de Urgencias (15), destacando que en los pacientes diabéticos sin CAD predominan las infecciones del tracto urinario mientras que en nuestra serie los dos procesos infecciosos asociados con mayor frecuencia a la CAD fueron las neumonías y las gastroenteritis aguda (16-19). La mortalidad de la CAD ha disminuido en las dos ultimas décadas, pasando del 30\% al 5$10 \%$ si bien en mayores de 50 años se habla de cifras entre $15-$ $25 \%(2-6,10,13,20)$, siendo en nuestra serie del $66 \%$ en este grupo de edad, que en parte se podría explicar debido a la presencia de procesos concomitantes graves en los mismos y la mayor edad media de la misma..Encontramos tres factores de mal pronóstico relacionados de forma significativa con la mortalidad: edad $>80, \mathrm{pH}<7,30$, glucemia $>700 \mathrm{mg} / \mathrm{dl}$, sin embargo esto no se refleja en otras series, donde sobre todo señalan como tales: la demora en el inicio del tratamiento, la depleción de agua y electrolitos, la presencia de cardiopatía isquémica no conocida previamente, no relacionándose la mortalidad por la presencia de cifras elevadas de glucemia o bajas de $\mathrm{pH}(1,20)$.

Queremos señalar como conclusiones: la edad de la CAD de nuestra serie es superior a la descrita en la literatura, la importante relación de la misma con la mortalidad, pudiéndose plantear que ya por sí solo podría ser criterio de ingreso en UCI. Las diferencias claras en cuanto a factores desencadenantes y forma de presentación entre los diabéticos conocidos y los no conocidos. Por último las diferencias entre los grupos de edad señalados, lo cual nos permite señalar que la CAD no es un problema circunscrito a pacientes jóvenes sino a todas las edades, debiendo los médicos de urgencias tener especial cuidado por su morbimortalidad en los ancianos.

\section{Bibliografía}

1. May M.E, Young C, King K. Resource utilization in treatment of Diabetic Ketoacidosis in adults. Am J Med Sci 1993; 306 (5): 287-294.

2. Snorgaard O, Eskildsen P.C, Vadstrup S, et al. Diabetic Ketoacidosis in Denmark: Epidemiology incidence rates, precipitating factors and mortality rates. J Intern Med 1989; 226: 223-228.

3. Moss JM: Diabetic Ketoacidosis: Effective low-cost treatment in a community hospital. South Med J 1987; 80: 875-881.

4. Tattersall R, Gregory R, Selby C, et al. Course of brittle diabetes: 12 year follow up. Br Med J 1991; 302: 1240-1243.

5. Ellemann K, Soerensen JN, Pedersen L, Edsberg B, Andersen OO. Epidemiology and treatment of Diabetic Ketoacidosis in a community population. Diabetes Care 1984; 7: 528-532

6. Faich GA, Fishbein HA, Ellis SE. The Epidemiology of diabetic acidosis: a populations-based study. Am J Epidemiol 1983; 117: 551-558.

7. Gouin P.E, Gossain VV, Rovner D.R. Diabetic Ketoacidosis: Outcome in a community hospital. South Med J 1985; 78: 941-943.

8. Walker M, Marshall S.M, Albert K.G. Clinical aspects of Diabetic Ketoacidosis. Diabetes Metab Rev 1989; 8: 651-663.

9. Kreisberg R.A. Diabetic Ketoacidosis, in Rifkin H, Porte D (eds): Diabetes Mellitus. Theory and Practice. New York, Elsevier 1990; 591-603.

10. Lester FT. Ketoacidosis in Ethiopian diabetics. Diabetología 1980; 18: 375-377.

11. Beigelman PM: Severe diabetic Ketoacidosis (“diabetic coma”): 482 episo- des in 257 patients; experience of three years. Diabetes 1971; 20: 490-500.

12. Morris W. Hospitalizations for patients with diabetes mellitus: Changing perspectives. Mayo Clin Proc 1990; 65: 1268-1271.

13. Green A, Hauge M, Holm N.V, Rasch LL. Epidemiological studies of diabetes mellitus in Denmark. Diabetologia 1981 II (20): 468-470.

14. Christian B, Kromann H, Ortved AO et al. Incidence, seasonal and geographical patterns of juvenile-onset insulin-dependent diabetes mellitus in Denmark. Diabetologia 1977; 13: 281-284.

15. Segado A, López-González C, Granda MJ, et al. Patología infecciosa en pacientes diabéticos atendidos en un departamento de urgencias. An Med Interna (Madrid) 1999; 16:3-7.

16. Smitherman KO, Peacock JE Jr. Infectious emergencies in patients with diabetes mellitus. Med Clin North Am 1995; 79 (1): 53-78.

17. Bagdad JD, Segreti J. The infections emergencies of diabetes. Endocrinologist 1991; 1: 155-162.

18. Wheat L.J. Infection and diabetes mellitus. Diabetes Care 1980; 3:187197.

19. Fekety R Jr, Caldwell J, Gump D et al. Bacteria, viruses and mycoplasma in acute pneumonia in adults. Am Rev Resp Dis 1971; 104: 499-507.

20. Tunbridge WMG. Factors contributing to deaths of diabetes under fifty years of age. Lancet 1981; 2: 569-572.

21. Smith DM, Weinberger M, Katz BP. Predicting nonelective hospitalisation: a model based on risk factors associated with diabetes mellitus. J Gen Intern Med 1987; 2: 168-173. 